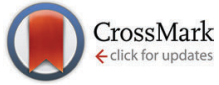

Cite this: Phys. Chem. Chem. Phys., 2015, 17, 23559

Received 10th July 2015 , Accepted 12th August 2015

DOI: $10.1039 / \mathrm{c} 5 \mathrm{cp} 04022 \mathrm{a}$

www.rsc.org/pccp

\title{
The surface roughness, but not the water molecular orientation varies with temperature at the water-air interface $\dagger$
}

\author{
Yuki Nagata, ${ }^{\star a}$ Taisuke Hasegawa, ${ }^{b}$ Ellen H. G. Backus, ${ }^{a}$ Kota Usui, ${ }^{a}$ \\ Seiji Yoshimune, ${ }^{a}$ Tatsuhiko Ohto ${ }^{c}$ and Mischa Bonn ${ }^{\star a}$
}

\begin{abstract}
We examine the temperature dependence of the interfacial molecular structure at the water-air interface by combining experimental and simulated sum-frequency generation (SFG) spectroscopy. The experimental SFG spectra of the $\mathrm{OH}$-stretching mode show a decrease in the amplitude at $\sim 3300 \mathrm{~cm}^{-1}$ with increasing temperature, while the $3700 \mathrm{~cm}^{-1}$ 'free $\mathrm{OH}^{\prime}$ SFG feature is insensitive to temperature changes. The simulated spectra are in excellent agreement with experiment. A comparison between interfacial SFG spectra and bulk infrared/Raman spectra reveals that the variation of the SFG signal due to the temperature change is not caused by a temperature-dependent $\mathrm{OH}$ bond orientation of the interfacial water molecules, but can be fully accounted for by the temperature dependence of the optical response of water. These results indicate that while the thickness of the interfacial region varies with temperature, the molecular organization of interfacial water at the water-air interface is surprisingly insensitive to temperature changes.
\end{abstract}

Temperature is a critical parameter of water which controls a variety of its macroscopic properties not only in the bulk but also at interfaces. The nature of interfacial water and its temperature dependence have been debated. ${ }^{1}$ It is well known, as first postulated by Faraday, that liquid water exists on the ice surface below the bulk freezing point. ${ }^{2,3}$ Furthermore, the surface tension of water is affected by temperature and decreases from $76 \mathrm{mN} \mathrm{m}^{-1}$ at $0{ }^{\circ} \mathrm{C}$ to $68 \mathrm{mN} \mathrm{m}^{-1}$ at $50{ }^{\circ} \mathrm{C} .{ }^{4}$ Although these observations must be related to the hydrogen bond $(\mathrm{HB})$ network at the water interface, questions remain regarding the temperature dependence of the interfacial molecular arrangement of water.

HB interactions in bulk water have been probed using various vibrational spectroscopic techniques such as infrared (IR) and Raman spectroscopy. ${ }^{5}$ Interfacial water molecules can be probed selectively with sum-frequency generation (SFG) spectroscopy. In this technique IR and visible laser pulses are combined at the interface and the sum-frequency is detected.

\footnotetext{
${ }^{a}$ Max Planck Institute for Polymer Research, Ackermannweg 10, 55128, Mainz, Germany.E-mail: nagata@mpip-mainz.mpg.de, bonn@mpip-mainz.mpg.de

${ }^{b}$ Department of Chemistry, Graduate School of Science, Kyoto University, Sakyoku, Kyoto 606-8502, Japan

${ }^{c}$ Graduate School of Engineering Science, Osaka University, 1-3 Machikaneyama, Toyonaka, Osaka, 560-8531, Japan

$\dagger$ Electronic supplementary information (ESI) available: MD simulation protocols, calculation of the SFG, IR, and Raman spectra, simulation results, and another experimental data. See DOI: 10.1039/c5cp04022a
}

Since this is a second order response, contributions from centrosymmetric media like bulk water are eliminated. With this technique, the interfacial water structure at the water-air interface has been studied and the presence of the dangling, free $\mathrm{OH}$ groups has been evidenced, arising from the interruption of the H-bonded network at the interface. , $^{6}$

Although this technique is very suitable to study the variations of the interfacial water structures with temperature change, only a limited number of studies have addressed these at the water-air ${ }^{6,8-10}$ and ice-air ${ }^{11-13}$ interfaces. At the $\mathrm{D}_{2} \mathrm{O}$-air interface, the OD stretch SFG features in the H-bonded region $\left(\sim 2300-2500 \mathrm{~cm}^{-1}\right)$ are reduced as well as blue-shifted with increasing temperature, while the peak intensities at the free OD stretch frequency $\left(\sim 2730 \mathrm{~cm}^{-1}\right)$ are insensitive to the temperature. From the enhancement of the H-bonded OD stretch SFG feature at the water-air interface as well as the water-hydrophobic material interfaces at lower temperatures, it was concluded that the ordering of the interfacial water structure is enhanced at reduced temperatures. ${ }^{9}$ It is, however, not a priori evident that the surface would become increasingly ordered at lower temperatures. Moreover, the optical response of water is known to vary with temperature; ${ }^{14}$ this effect has not been considered.

Notably, the second-harmonic generation (SHG) intensity from the water-air interface ${ }^{15-17}$ has been reported to be rather insensitive to the temperature (at most by $10 \%$ over a $70 \mathrm{~K}$ temperature range), ${ }^{16}$ which seems at odds with the $40 \%$ variation in the 
hydrogen-bonded OH stretch signal in the SFG spectra over a $40 \mathrm{~K}$ temperature range. ${ }^{9}$ However, since the SHG intensity arises from the electronic transition dipole moment of the interfacial water molecules, while the SFG signals arise from the vibrational transition dipole moment, the SHG intensity cannot probe the free $\mathrm{OH}$ and hydrogen-bonded $\mathrm{OH}$ groups separately. It is thus challenging to make a direct connection between $\mathrm{OH}$ bond orientation and temperature-dependent SHG results, whereas SFG, with its molecular specificity, in principle provides such information.

In addition to fundamental interest in the temperature dependence of the interfacial water structure, understanding the temperature dependent SFG spectra is essential to interpret the pump/probe or two-dimensional SFG data. ${ }^{18-24}$ In these time-resolved techniques, a specific vibrational mode is excited and the fate of the excitation, e.g. depolarization, spectral diffusion, and vibrational relaxation, is followed in time through transient changes in the SFG spectra. In the course of time - typically a few picoseconds - the excess vibrational energy is transferred to heat. The time-dependent increase in the surface temperature thus also gives rise to non-zero differential SFG spectra. Hence, knowledge on the variation of the SFG spectra with temperature is needed to disentangle the contribution of the interfacial water dynamics to the transient spectra from the appearance of heat. ${ }^{25}$

In order to explore the effect of temperature on the microscopic structure of the interfacial water and the SFG spectra, we measure the heterodyne-detected SFG (HD-SFG) signals at the water-air interface at ssp polarization and compute the corresponding spectra by using MD simulation with different temperatures. Here, ssp polarization denotes s-polarized SFG, s-polarized visible, and p-polarized IR beams, respectively. Both simulated and experimentally measured $\mathrm{OH}$ stretch SFG spectra show a dramatic reduction of the $3250-3350 \mathrm{~cm}^{-1}$ $\mathrm{OH}$ stretch band and unchanged amplitude of the free $\mathrm{OH}$ $\left(\sim 3700 \mathrm{~cm}^{-1}\right)$ stretch peak with increasing temperature. We subsequently compare the variation of the interfacial SFG signals with that of the bulk IR/Raman spectra in the simulation, revealing that the temperature dependence of the SFG spectra arises primarily from the variation in the optical response rather than from changes in the interfacial structure. We find that the $\mathrm{OH}$ group orientation of the interfacial water molecules is remarkably insensitive to the temperature.

Experimentally, $\mathrm{HD}$-SFG spectra of the $\mathrm{H}_{2} \mathrm{O}$-air interface were measured in a temperature controlled metal trough at $283 \pm 1$ and $303 \pm 1 \mathrm{~K}$. Part of a Ti:Sapphire based regenerative amplifier (Spitfire Ace, Spectra-Physics) was used to pump a TOPAS (Light Conversion) generating broadband IR pulses centered at $\sim 3300 \mathrm{~cm}^{-1}$ with a full width at half maximum (FWHM) of $400 \mathrm{~cm}^{-1}$. Another part of the laser output was sent through an elaton to obtain narrowband visible pulses around $801 \mathrm{~nm}$ with a FWHM of $\sim 25 \mathrm{~cm}^{-1}$. The IR and visible beams were first overlapped on the surface of a gold mirror to generate the first SFG signal under almost grazing incidence angle (IR and visible incident angle: $\sim 85$ and $75^{\circ}$, respectively). Subsequently the IR and visible pulses and the SFG signal were reflected on a concave mirror with a focal length of $50 \mathrm{~mm}$ to the sample, with an angle of incidence of $\sim 45$ and $35^{\circ}$ for the IR and visible beams, respectively. After collimation the two SFG signals are sent to the detector where they interfere. The spectra were normalized to the SFG signal from $z$-cut quartz. The depicted spectra were averaged for two 5 minutes exposures and the height was corrected in between using a height sensor with a resolution of $200 \mathrm{~nm}$ (Keyence, LK-G85). The phase changes due to evaporation were less than 10 and $30^{\circ}$ at 283 and $303 \mathrm{~K}$, respectively. Following ref. 26 the data were rephased using the SFG data of the $\mathrm{D}_{2} \mathrm{O}$-air interface measured at both temperatures and thus undergoing the same phase drift due to evaporation as the $\mathrm{H}_{2} \mathrm{O}$ measurements. Therefore, we estimate that the phase uncertainty in the final spectra is less than $10^{\circ}$ for both temperatures. Spectra for additional temperatures can be found in the ESI $\dagger$, as well as the spectra corrected for the temperature dependent Fresnel factors.

MD simulations with the $a b$ initio based force field model (melting temperature of $265-270 \mathrm{~K}$, see ESI $\dagger$ ) at the water-air interface were performed at temperatures of 282, 293, 305, 318, and $331 \mathrm{~K}$. The dipole moment and polarizability can be readily computed with this force field model, ${ }^{27,28}$ and the SFG signals were calculated from the simulated dipole moment and polarizability within the truncating response function formalism. ${ }^{28,29}$ Note that these $a b$ initio based force field models have been actively developed by several groups. ${ }^{30-33}$ In this formalism, the relevant time correlation function is calculated in a manner that allows for delocalization of the vibrational amplitude over several chromophores. The spatial extent over which vibrational delocalization is allowed in the calculations is given by the cutoff radius $r_{\mathrm{t}}$. The Fourier transform of the time correlation function into the frequency domain provides access to the SFG response. The MD simulation protocols, the SFG signal calculation, and the dependence of the SFG spectra on the value of $r_{\mathrm{t}}$ are provided in the ESI. $\dagger$ Furthermore, the temperature dependences of the self-diffusion constant in the bulk and surface tension at the water-air interface are given in the ESI. $\dagger$

The SFG response of the $\mathrm{OH}$ stretch mode was fully converged with $r_{\mathrm{t}}=6 \AA$ (including cross-correlation within the $6 \AA$ cutoff sphere, see the ESI $\dagger$ ). The comparison of the simulated and measured SFG spectra is presented in Fig. 1, which reveals good agreement between simulation and experiment: the amplitude at $\sim 3700 \mathrm{~cm}^{-1}$ is rather insensitive to the temperature, while the amplitude at $\sim 3300 \mathrm{~cm}^{-1}$ decreases significantly with increasing temperature. We focus here on the 3150 to $3750 \mathrm{~cm}^{-1}$ frequency range as the assignment of the bands in the spectrum is unambiguous in this range. ${ }^{7,26}$ In addition, the MD simulation indicates that the positive shoulder at $\sim 3600 \mathrm{~cm}^{-1}$ becomes more prominent with decreasing temperature. This positive shoulder, also apparent in the experiment, has been assigned to the anti-symmetric stretch mode of the interfacial water molecules donating two HBs. $^{34}$ The enhanced amplitude for this anti-symmetric mode indicates that the frequencies of the two $\mathrm{OH}$ stretch modes in each water molecule overlap more strongly with decreasing temperature, giving rise to larger vibrational energy splitting. The thermal 


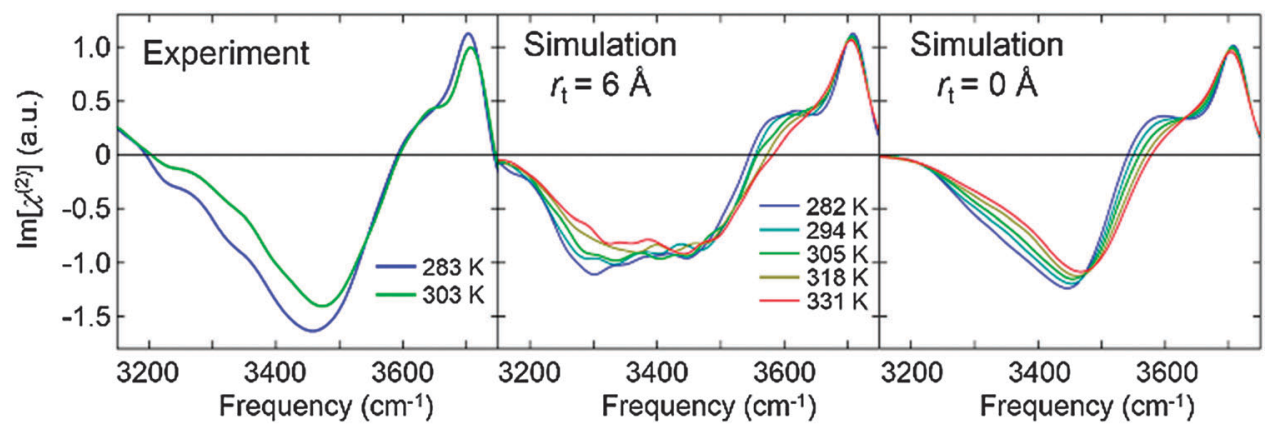

Fig. 1 Simulated SFG spectra of the OH stretching frequency with different truncating distances $r_{\mathrm{t}}=0$ and $6 \AA$ and experimental ssp SFG spectra. The SFG signals were normalized at the free OH stretch peak of the SFG spectra with $r_{\mathrm{t}}=0 \AA$ at $305 \mathrm{~K}$ for the simulation and at $303 \mathrm{~K}$ for the experiment.

variation of the $\sim 3600 \mathrm{~cm}^{-1}$ feature is not so prominent in the experimental HD-SFG spectra due to limited signal-to-noise above $3600 \mathrm{~cm}^{-1}$. In any case, our MD simulations capture the temperature dependence of the SFG spectra very well.

To investigate the temperature dependence of the vibrational delocalization for $\chi^{(2)}$ spectra, we also calculated the variation of $\operatorname{Im}\left[\chi^{(2)}\right]$ with $r_{\mathrm{t}}=0 \AA$ (without cross-correlation), which is also plotted in Fig. 1. Clearly, cross-correlation terms enhance the negative $\sim 3300 \mathrm{~cm}^{-1}$ feature dramatically. Since the cross-correlation terms allow intermolecular coupling to contribute to the spectra, this peak enhancement arises from the $\mathrm{H}_{2} \mathrm{O}-\mathrm{H}_{2} \mathrm{O}$ vibrational coupling. The observation of the enhanced amplitude at the red side in the presence of intermolecular coupling is in good agreement with the SFG measurement using the neat $\mathrm{H}_{2} \mathrm{O}$ and isotopically diluted $\mathrm{H}_{2} \mathrm{O}$ (see Fig. 2 of ref. 26). Surprisingly, also without the intermolecular coupling $\left(r_{\mathrm{t}}=0 \AA\right)$, we can see a clear reduction of the SFG response at $\sim 3300 \mathrm{~cm}^{-1}$ with increasing temperature. This indicates that the intermolecular coupling does not play an essential role in the variation of the SFG amplitude with temperatures. Here, it should be noted that although the intermolecular couplings of the $\mathrm{OH}$ stretch chromophores can be controlled with the truncation of the response function, intermolecular coupling effects are inevitably included through the induced molecular dipole moment and polarizability.

We now explore the mechanism of the $\sim 3300 \mathrm{~cm}^{-1}$ SFG amplitude variation due to the temperature change. The SFG amplitude is determined by (1) the optical properties such as the transition dipole moment, the transition polarizability, and the decay of the dipole and polarizability time correlation functions and (2) the number of the ordered water molecules and the degree of order. ${ }^{35,36}$ To conclude that the reduction of the SFG signal arises from the less ordered structure of interfacial water (2), it is required that the temperature dependence of the optical properties (1) is negligible. To examine the temperature dependence of the optical responses by excluding the effects of the ordered water structure, we estimated the variation of the optical responses of the transition dipole moment and polarizability from the IR and Raman responses. Note that the IR and Raman responses arise from the timecorrelation functions of transition dipole moment and polarizability, respectively. ${ }^{37}$ Similar analyses have been made in ref. 38 and 39. We simulated the IR ( $\left.I_{\mathrm{IR}}\right)$ and VV Raman signals $\left(I_{\text {Raman }}\right)$ for bulk water with different temperatures and constructed the $\sqrt{I_{\mathrm{IR}} I_{\text {Raman }}}$ spectra. Note that since $\operatorname{Im}\left[\chi_{\mathrm{ssp}}{ }^{(2)}\right]$ is compared with the $\sqrt{I_{\mathrm{IR}} I_{\mathrm{Raman}}}$ spectra, we adopted the $\mathrm{VV}$ Raman spectra rather than VH Raman spectra. ${ }^{40}$ The simulated spectra are shown in Fig. 2. The temperature dependence of the IR and Raman spectra for the $\mathrm{OH}$ stretching mode are consistent with experimental data from literature; ${ }^{41,42}$ the IR intensity decreases in the frequency range of $3100 \mathrm{~cm}^{-1}<\omega<3550 \mathrm{~cm}^{-1}$ with increasing temperature, while the VV Raman spectra shows a dramatic change in a narrower frequency region $\left(3100 \mathrm{~cm}^{-1}<\right.$ $\omega<3350 \mathrm{~cm}^{-1}$ ).

Subsequently, we compared the temperature variation of the $\sqrt{I_{\mathrm{IR}} I_{\text {Raman }}}$ amplitudes with that of the simulated $\operatorname{Im}\left[\chi^{(2)}\right]$ amplitudes. The left panel of Fig. 3 displays the correlation map of their spectral areas integrated in the region

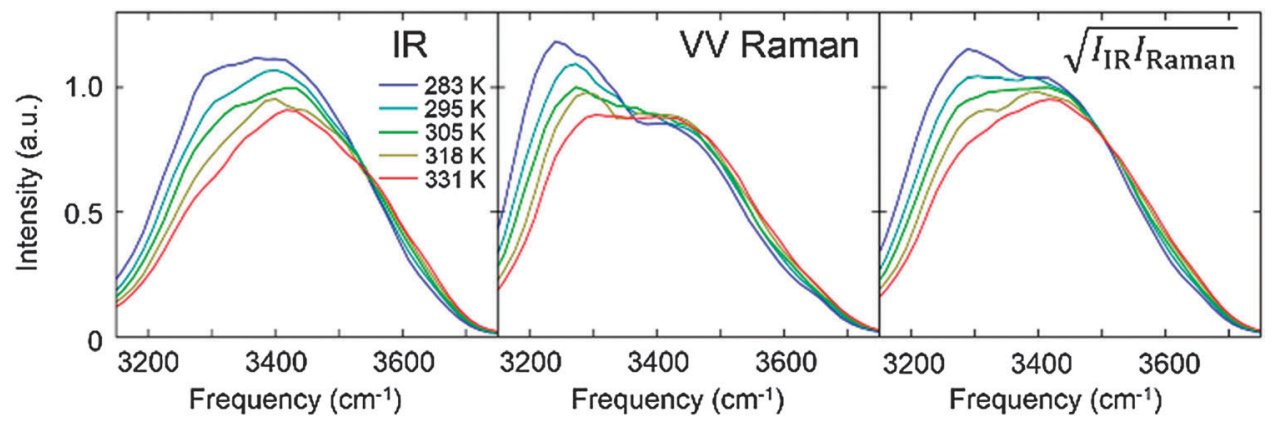

Fig. 2 Simulated IR, VV Raman, and $\sqrt{I_{\mathrm{IR}} I_{\text {Raman }}}$ spectra in the $\mathrm{OH}$ stretching frequency range. 

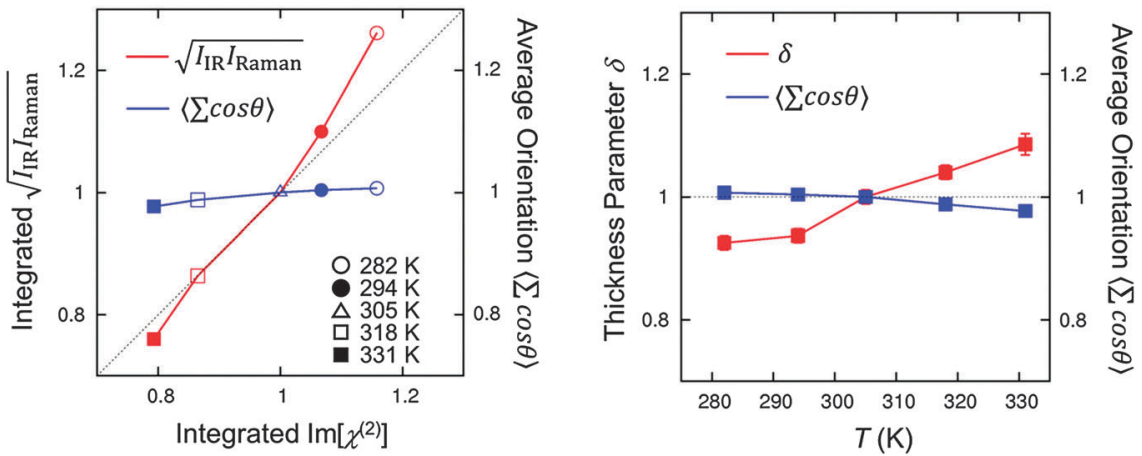

Fig. 3 (left) Correlation map of the spectral area $\operatorname{Im}\left[\chi^{(2)}\right]$ integrated for $3250 \mathrm{~cm}^{-1}<\omega<3350 \mathrm{~cm}^{-1}$ vs. the integrated $\sqrt{I_{\mathrm{IR}} I_{\mathrm{Raman}}}$ spectra in the same frequency range and the average orientation of the $\mathrm{H}$-bonded $\mathrm{OH}$ groups $\langle\Sigma \cos \theta\rangle$. (right) Temperature dependences of $\langle\Sigma \cos \theta\rangle$ and thickness parameter $\delta$. All the data were normalized to the value of the data at $305 \mathrm{~K}$.

of $3250 \mathrm{~cm}^{-1}<\omega<3350 \mathrm{~cm}^{-1}$, i.e. the region of the largest change. This figure shows a very clear correlation between the changes in the SFG response and changes in the $\sqrt{I_{\mathrm{IR}} I_{\mathrm{Raman}}}$ spectra, implying that the large reduction of the SFG amplitudes with increasing temperature does not arise from the less ordered $\mathrm{OH}$ bonds, but can be fully accounted for by taking into consideration the temperature dependence of the optical properties of water. The origin of the decrease in the temperaturedependent optical response with increasing temperature, is mainly due to a speed-up of the dephasing of the molecular vibrations, and due to weakening of the H-bonded network.

To independently confirm that the variation of the SFG spectra with temperature is not caused by changes in the ordering of the $\mathrm{OH}$ groups, we compared the integrated $\operatorname{Im}\left[\chi^{(2)}\right]$ amplitude with the simulated total orientations of the $\mathrm{H}$-bonded $\mathrm{OH}$ groups per unit area $\langle\Sigma \cos \theta\rangle$, where $\theta$ is the angle formed by the surface normal and the $\mathrm{H}$-bonded $\mathrm{OH}$ group. The calculation details are given in the ESI. $\dagger$ The lack of correlation between the temperature dependence of $\operatorname{Im}\left[\chi^{(2)}\right]$ and average orientation is evident from the left panel of Fig. 3, clearly illustrating that the variation of the H-bonded $\mathrm{OH}$ stretch feature in the $\operatorname{Im}\left[\chi^{(2)}\right]$ spectra is little, if at all, affected by changes in the structure. Indeed, the orientation of the interfacial $\mathrm{OH}$ groups with the $\mathrm{HB}$ donor is almost unchanged over the temperature range of $280-330 \mathrm{~K}$.

While the average $\mathrm{H}$-bonded $\mathrm{OH}$ bond orientation of the interfacial water molecules is insensitive to the temperature, the thermal excitation of capillary waves should affect the interface. To quantify this, we calculated the thickness parameter $\delta$ by fitting the function

$$
\rho(z)=a\left(1-\tanh \left(-\frac{z-z_{\mathrm{G}}}{\delta}\right)\right)
$$

to the density profile $\rho(z)$ along the surface normal, where $z_{\mathrm{G}}$ is the position of the Gibbs dividing surface and $a$ is the half of the bulk density. The details can be found in the ESI. $\dagger$ The thickness $\delta$, along with the orientational average, $v s$. temperature is plotted in the right panel of Fig. 3. This clearly shows that, unlike the $\mathrm{OH}$ bond orientation, the surface roughness varies substantially with temperature. Here, we would like to note that the interfacial region with the thickness of $\delta$ is not the same as the SFG active region. An SFG signal originates from the interfacial region where the net dipole moment along the surface normal does not vanish.

In this paper, we investigated the molecular organization of the interfacial water molecules at the water-air interface and found that the orientation of the water molecules $(\langle\Sigma \cos \theta\rangle)$ does not change with temperature, whereas the surface roughness $(\delta)$ varies. Furthermore, we also reproduced the temperature dependence of the surface tension (see ESI $\dagger$ ). It would be important to note that the interfacial region which has the net orientation and less density than the bulk is not the same as the region which affects the surface tension; the surface tension has been determined by the interfacial water organization on larger length scale than the region which the SFG probes. There is thus no inconsistency between no temperature dependence of $\langle\Sigma \cos \theta\rangle$ and the temperature-dependent surface tension.

In conclusion, we investigated the temperature dependence of the SFG spectrum at the water-air interface using a combined experimental and simulated SFG approach. For the $\mathrm{OH}$ stretch mode, the SFG amplitude at $\sim 3700 \mathrm{~cm}^{-1}$ is insensitive to the temperature, while the amplitude at $\sim 3300 \mathrm{~cm}^{-1}$ decreases with increasing temperature. By comparing the $\sim 3300 \mathrm{~cm}^{-1}$ peak enhancement in $\operatorname{Im}\left[\chi^{(2)}\right]$ with the $\sqrt{I_{\mathrm{IR}} I_{\text {Raman }}}$ spectra and the average orientations, we conclude that the variation of the SFG amplitude at $\sim 3300 \mathrm{~cm}^{-1}$ due to the temperature change is the result of the temperature dependence of the optical response. Surprisingly, the $\mathrm{OH}$ bond orientation of the interfacial water molecules is largely insensitive to the temperature, despite the substantial change of the SFG response with temperature. Our study illustrates that care must be taken when interpreting temperature-dependent SFG signals in terms of temperature-dependent interfacial structure. This understanding is particularly relevant to the study of temperaturedependent ordered water structures at for example hydrophobic or biological interfaces such as anti-freezing protein-water interface. ${ }^{9,43}$ Conclusions based on temperature dependent SFG spectra drawn previously might have to be re-examined by comparing the temperature dependence of the IR and Raman intensity with the SFG spectra. 


\section{Conflict of interest}

The authors declare no competing financial interests.

\section{Acknowledgements}

We thank Dr Johannes Hunger, Dr Grazia Gonella, Prof. Eric Tyrode, Prof. Chuanshan Tian, and Prof. Markus Mezger for fruitful discussions. YN acknowledges the financial support from the German Science Foundation through the TRR146.

\section{References}

1 P. Jungwirth and D. J. Tobias, Molecular Structure of Salt Solutions: A New View of the Interface with Implications for Heterogeneous Atmospheric Chemistry, J. Phys. Chem. B, 2001, 105, 10468-10472.

2 A. Döppenschmidt and H.-J. Butt, Measuring the Thickness of the Liquid-like Layer on Ice Surfaces with Atomic Force Microscopy, Langmuir, 2000, 16, 6709-6714.

3 R. Rosenberg, Why Is Ice Slippery?, Phys. Today, 2005, 58, 50-54.

4 N. B. Vargaftik, B. N. Volkov and L. D. Voljak, International Tables of the Surface Tension of Water, J. Phys. Chem. Ref. Data, 1983, 12, 817-820.

5 H. J. Bakker and J. L. Skinner, Vibrational Spectroscopy as a Probe of Structure and Dynamics in Liquid Water, Chem. Rev., 2010, 110, 1498-1517.

6 Q. Du, R. Superfine, E. Freysz and Y. Shen, Vibrational Spectroscopy of Water at the Vapor/water Interface, Phys. Rev. Lett., 1993, 70, 2313-2316.

7 C. Tian and Y. R. Shen, Isotopic Dilution Study of the Water/ vapor Interface by Phase-Sensitive Sum-Frequency Vibrational Spectroscopy, J. Am. Chem. Soc., 2009, 131, 2790-2791.

8 C. Schnitzer, S. Baldelli and M. J. Shultz, Sum Frequency Generation by Water on Supercooled $\mathrm{H}_{2} \mathrm{SO}_{4} / \mathrm{H}_{2} \mathrm{O}$ Liquid Solutions at Stratospheric Temperature, Chem. Phys. Lett., 1999, 313, 416-420.

9 S. Strazdaite, J. Versluis, E. H. G. Backus and H. J. Bakker, Enhanced Ordering of Water at Hydrophobic Surfaces, J. Chem. Phys., 2014, 140, 054711.

10 M. Bonn, Y. Nagata and E. H. G. Backus, Molecular Structure and Dynamics of Water at the Water-Air Interface Studied with Surface-Specific Vibrational Spectroscopy, Angew. Chem., Int. Ed., 2015, 54, 5560-5576.

11 X. Wei, P. B. Miranda and Y. R. Shen, Surface Vibrational Spectroscopic Study of Surface Melting of Ice, Phys. Rev. Lett., 2001, 86, 1554-1557.

12 H. Groenzin, I. Li, V. Buch and M. J. Shultz, The SingleCrystal, Basal Face of Ice $I_{h}$ Investigated with Sum Frequency Generation, J. Chem. Phys., 2007, 127, 214502.

13 T. Ishiyama, H. Takahashi and A. Morita, Origin of Vibrational Spectroscopic Response at Ice Surface, J. Phys. Chem. Lett., 2012, 3, 3001-3006.
14 S. A. Corcelli and J. L. Skinner, Infrared and Raman Line Shapes of Dilute HOD in Liquid $\mathrm{H}_{2} \mathrm{O}$ and $\mathrm{D}_{2} \mathrm{O}$ from 10 to $90{ }^{\circ} \mathrm{C}, \mathrm{J}$. Phys. Chem. A, 2005, 109, 6154-6165.

15 M. C. Goh and K. B. Eisenthal, The Energetics of Orientation at the Liquid-Vapor Interface of Water, Chem. Phys. Lett., 1989, 157, 101-104.

16 W. K. Zhang, D. S. Zheng, Y. Y. Xu, H. T. Bian, Y. Guo and H. F. Wang, Reconsideration of Second-Harmonic Generation from Isotropic Liquid Interface: Broken Kleinman Symmetry of Neat Air/water Interface from Dipolar Contribution, J. Chem. Phys., 2005, 123, 224713.

17 W. Zhang, H. Wang and D. Zheng, Quantitative Measurement and Interpretation of Optical Second Harmonic Generation from Molecular Interfaces, Phys. Chem. Chem. Phys., 2006, 8, 4041-4052.

18 J. A. McGuire and Y. R. Shen, Ultrafast Vibrational Dynamics at Water Interfaces, Science, 2006, 313, 1945-1948.

19 A. Ghosh, M. Smits, J. Bredenbeck and M. Bonn, MembraneBound Water Is Energetically Decoupled from Nearby Bulk Water: An Ultrafast Surface-Specific Investigation, J. Am. Chem. Soc., 2007, 129, 9608-9609.

20 J. Bredenbeck, A. Ghosh, M. Smits and M. Bonn, Ultrafast Two Dimensional-Infrared Spectroscopy of a Molecular Monolayer, J. Am. Chem. Soc., 2008, 130, 2152-2153.

21 A. Eftekhari-Bafrooei and E. Borguet, Effect of HydrogenBond Strength on the Vibrational Relaxation of Interfacial Water, J. Am. Chem. Soc., 2010, 132, 3756-3761.

22 J. E. Laaser, W. Xiong and M. T. Zanni, Time-Domain SFG Spectroscopy Using Mid-IR Pulse Shaping: Practical and Intrinsic Advantages, J. Phys. Chem. B, 2011, 115, 2536-2546.

23 P. C. Singh, S. Nihonyanagi, S. Yamaguchi and T. Tahara, Communication: Ultrafast Vibrational Dynamics of Hydrogen Bond Network Terminated at the Air/water Interface: A Two-Dimensional Heterodyne-Detected Vibrational Sum Frequency Generation Study, J. Chem. Phys., 2013, 139, 161101.

24 C.-S. Hsieh, M. Okuno, J. Hunger, E. H. G. Backus, Y. Nagata and M. Bonn, Aqueous Heterogeneity at the Air/water Interface Revealed by 2D-HD-SFG Spectroscopy, Angew. Chem., Int. Ed. Engl., 2014, 53, 8146-8149.

25 K. Ramasesha, L. De Marco, A. Mandal and A. Tokmakoff, Water Vibrations Have Strongly Mixed Intra- and Intermolecular Character, Nat. Chem., 2013, 5, 935-940.

26 S. Nihonyanagi, T. Ishiyama, T.-K. Lee, S. Yamaguchi, M. Bonn, A. Morita and T. Tahara, Unified Molecular View of the Air/water Interface Based on Experimental and Theoretical $\chi^{(2)}$ Spectra of an Isotopically Diluted Water Surface, J. Am. Chem. Soc., 2011, 133, 16875-16880.

27 T. Hasegawa and Y. Tanimura, A Polarizable Water Model for Intramolecular and Intermolecular Vibrational Spectroscopies, J. Phys. Chem. B, 2011, 115, 5545-5553.

28 Y. Nagata, C.-S. Hsieh, T. Hasegawa, J. Voll, E. H. G. Backus and M. Bonn, Water Bending Mode at the Water-Vapor Interface Probed by Sum-Frequency Generation Spectroscopy: A Combined Molecular Dynamics Simulation and Experimental Study, J. Phys. Chem. Lett., 2013, 4, 1872-1877. 
29 Y. Nagata and S. Mukamel, Vibrational Sum-Frequency Generation Spectroscopy at the Water/Lipid Interface: Molecular Dynamics Simulation Study, J. Am. Chem. Soc., 2010, 132, 6434-6442.

30 Y. Wang and J. M. Bowman, Ab Initio Potential and Dipole Moment Surfaces for Water. II. Local-Monomer Calculations of the Infrared Spectra of Water Clusters, J. Chem. Phys., 2011, 134, 154510.

31 J. M. Bowman, Y. Wang, H. Liu and J. S. Mancini, Ab Initio Quantum Approaches to the IR Spectroscopy of Water and Hydrates, J. Phys. Chem. Lett., 2015, 6, 366-373.

32 V. Babin, C. Leforestier and F. Paesani, Development of a "First Principles" Water Potential with Flexible Monomers: Dimer Potential Energy Surface, VRT Spectrum, and Second Virial Coefficient, J. Chem. Theory Comput., 2013, 9, 5395-5403.

33 G. R. Medders and F. Paesani, Infrared and Raman Spectroscopy of Liquid Water through "First Principles" ManyBody Molecular Dynamics, J. Chem. Theory Comput., 2015, 11, 1145-1154.

34 I. V. Stiopkin, C. Weeraman, P. A. Pieniazek, F. Y. Shalhout, J. L. Skinner and A. V. Benderskii, Hydrogen Bonding at the Water Surface Revealed by Isotopic Dilution Spectroscopy, Nature, 2011, 474, 192-195.

35 A. Morita and J. T. Hynes, A Theoretical Analysis of the Sum Frequency Generation Spectrum of the Water Surface, Chem. Phys., 2000, 258, 371-390.
36 B. M. Auer and J. L. Skinner, Vibrational Sum-Frequency Spectroscopy of the Liquid/vapor Interface for Dilute HOD in $\mathrm{D}_{2} \mathrm{O}, \mathrm{J}$. Chem. Phys., 2008, 129, 214705.

37 R. W. Boyd, Nonlinear Optics, Academic Press, Waltham, 2003.

38 E. L. Hommel and H. C. Allen, The Air-Liquid Interface of Benzene, Toluene, M-Xylene, and Mesitylene: A Sum Frequency, Raman, and Infrared Spectroscopic Study, Analyst, 2003, 128, 750-755.

39 S. Gopalakrishnan, P. Jungwirth, D. J. Tobias and H. C. Allen, Air-Liquid Interfaces of Aqueous Solutions Containing Ammonium and Sulfate: Spectroscopic and Molecular Dynamics Studies, J. Phys. Chem. B, 2005, 109, 8861-8872.

40 B. M. Auer and J. L. Skinner, IR and Raman Spectra of Liquid Water: Theory and Interpretation, J. Chem. Phys., 2008, 128, 224511.

41 J. R. Scherer, M. K. Go and S. Kint, Raman Spectra and Structure of Water from -10 to $90^{\circ}, \mathrm{J}$. Phys. Chem., 1974, 78, 1304-1313.

42 Y. Maréchal, Infrared Spectra of Water. I. Effect of Temperature and of H/D Isotopic Dilution, J. Chem. Phys., 1991, 95, 5565.

43 K. Meister, S. Strazdaite, A. L. Devries, S. Lotze, L. L. C. Olijve, I. K. Voets and H. J. Bakker, Observation of Ice-like Water Layers at an Aqueous Protein Surface, Proc. Natl. Acad. Sci. U. S. A., 2014, 111, 17732-17736. 